\title{
A Case of Peritoneal-Pelvic Tuberculosis With Elevated CA-125; An Enigma as Ovarian Cancer
}

\section{Prakash Raj Oli1, Rosy Vaidya Malla ${ }^{2}$ and Kavita Karmacharya ${ }^{3}$}

${ }^{1}$ Intern, Nepalese Army Institute of Health Sciences, Shree Birendra Hospital, Chhauni, Kathmandu, Nepal.

${ }^{2}$ Department of Gynecology and Obstetrics, Nepalese Army Institute of Health Sciences, Shree Birendra Hospital, Chhauni, Kathmandu, Nepal.

${ }^{3}$ Department of Pathology, Nepalese Army Institute of Health Sciences, Shree Birendra Hospital, Chhauni, Kathmandu, Nepal.

\section{ABSTRACT}

Peritoneal-pelvic tuberculosis is a rare form of extrapulmonary-TB mainly affecting women of 20 to 40 years, especially in TB endemic countries. It classically presents with abdominal pain, menstrual irregularities, adnexal mass, and elevated serum CA-125 level, creating confusion with genital malignancy, especially ovarian, leading to difficulty in its management and often leads to devastating surgeries. Here a case of peritoneal-pelvic TB, a young lady with abdominal pain, radiologic ascites and adnexal mass, and elevated serum CA-125 level is presented. So, it should always be one of the differential diagnosis of ovarian cancer especially among young women in TB endemic countries.

Key words: CA-125; Nepal; Ovarian Cancer; Peritoneal-Pelvic Tuberculosis

Correspondence: Prakash Raj Oli. Intern. Nepalese Army Institute of Health Sciences, Shree Birendra Hospital, Chhauni, Kathmandu, Nepal. Email: rajoliprakash@gmail.com

DOI: http://dx.doi.org/10.3126/mjsbh.v18i2.22390

Submitted on: 2019-01-19

Accepted on: 2019-06-11

This work is licensed under creative common license:

http://creativecommons.org/licenses/by-nc-nd/4.0/ C MJSBH 2019 


\section{INTRODUCTION}

During 2016/17 AD, a total of 31,764 TB cases, $29 \%$ being extrapulmonary-TB, were reported from Nepal. ${ }^{1}$ Among different forms of extrapulmonaryTB, Peritoneal-pelvic Tuberculosis is rare-type with unknown true incidence due to its atypical presentation. ${ }^{2}$ Despite its classic presentation of abdominal pain, menstrual disturbances, pelvic masses, and elevated serum CA-125 level, none of these features are specific for its diagnosis and differentiation from genitourinary malignancy, especially ovarian, among adult women. ${ }^{3,4}$ So, its diagnosis requires a high level of suspicion among adult women presenting with such features in TB endemic region and prevents inappropriate management of patient. ${ }^{4}$

\section{CASE REPORT}

A 38 years $\mathrm{P}_{1+2} \mathrm{~L}_{1}$ lady presented with the intermittent dull-aching type of lower abdominal pain for four months duration and had a family history of TB in grandfather. On physical examination, only significant finding was a presence of fullness of left fornix with a normalsized uterus on a per-vaginal examination.

All routine investigations, including chest $\mathrm{x}$-ray, were normal. USG-abdomen showed an enlarged right ovary of approximately $5.4 \times 4.4 \times 3.4 \mathrm{~cm}$ with an irregular outline and a small cystic area and moderate ascites. CECT-abdomen/pelvis revealed an irregularly marginated cystic/solid complex right adnexal cyst of $5.4 \times 3.8 \times 9.2 \mathrm{~cm}$ size, multiple enlarged retroperitoneal lymph nodes having areas of necrosis, omental thickening, and moderate ascites. Serum CA-125 level was elevated to 426.0 $\mathrm{U} / \mathrm{ml}$. With these findings, the patient was planned for explorative laparotomy with suspicion of ovarian malignancy. Laparotomy revealed about $200 \mathrm{ml}$ of straw-colored ascitic fluid in peritoneal cavity which was sent for its analysis including AFB staining, an enlarged left ovary of approximately $6 \times 5 \mathrm{~cm}$ with multiple miliary deposits. Military deposits also were seen over bilateral fallopian tubes and ovaries, whole uterine surface, lateral pelvic wall, intestine, thickened omentum, under the surface of liver and bladder. Then, suspicious left fallopian tube and ovary were resected along with a piece of parietal peritoneum and sent for histopathological examination.

Histopathological examination revealed noncaseating granulomas in the fallopian tube, ovary, and peritoneum with no neoplastic pathology and $\mathrm{AFB}$ on $\mathrm{ZN}$-staining of tissue. The ascitic fluid study was negative for malignant cells and AFB.

Finally, a post-operative diagnosis of peritonealpelvic tuberculosis was made and the patient was relieved of malignancy-related psychological stress and enrolled into DOTS.

\section{DISCUSSION}

Peritoneal-pelvic TB is a subacute disease and usually develops secondary to haematogenous spread of primary pulmonary infection which usually heals completely without any clinical or radiological residues or from rupture of tubercular mesenteric lymph nodes. ${ }^{5}$ It occurs frequently in women of 14 to 72 years, mostly of 20 to 40 years. ${ }^{2,6}$ It classically presents with abdominal pain
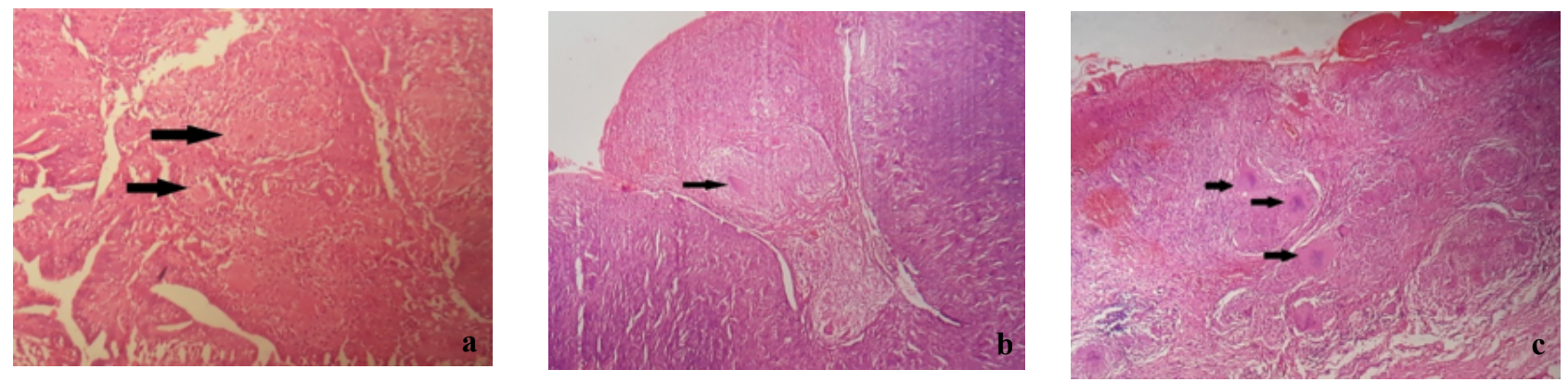

Figure 1. Non-caseating granulomas (arrows) with no malignant cells in the specimen from: (a) Fallopian tube, (b) Ovary and (c) Peritoneum. 
( $45-100 \%$ ), menstrual disturbance (oligomenorrhoea) (4-54\%), polymenorrhoea (26.9\%), dysmenorrhea (12-30\%), menorrhagia (19\%), hypomenorrhoea (15.4\%), amenorrhea (14\%), infertility (43-74\%), abdominal distention (30.7-45\%), weight loss (20-40\%), abdominal mass (19.2-35\%), anorexia (15-20\%), fever (7.7-30\%), positive past history/family history of TB (10-85\%), night sweats $(9.09 \%)$, diarrhoea $(5 \%)$, urinary frequency $(5 \%)$, dyspnea $(5 \%))^{2,6-8}$

Ultrasonography and CT scan have been used for its diagnosis but have limited efficacy due to its diffuse nature and small implants. Ultrasound usually shows exudative ascites-free or partitioned, thickened peritoneum, clumping of bowel loops to each other or to the anterior abdominal wall, peritoneal nodules, enlarged lymph nodes, and adnexal masses. On CT scan, high density (25 to 45 HU) ascites, enlarged lymph nodes with a hypodense centre and hyperdense rim or often calcified, thickened mesentery and omentum, uniformly thickened peritoneum and clumped bowel loops may present. ${ }^{5}$

Any woman with a radiologically identifiable pelvic mass, ascites and raised CA-125 levels is normally considered to have ovarian cancer unless proved otherwise considering its serious nature. ${ }^{10}$ Since patient presented with an atypical symptom, radiologic adnexal mass, and elevated serum CA-125, she was suspected of having ovarian cancer preoperatively and hence a laparotomy had been performed.

Due to low sensitivity and the fact that CA-125 also is produced by normal epithelial cells including of the peritoneum, pleura, and pericardium when inflamed should prompt consideration of nononcological causes such as infections, tuberculosis, endometriosis, menstruation, pregnancy, and a number of non-gynaecologic conditions like active hepatitis, pneumonia, especially in presence of serosal fluid among young women. ${ }^{3,6}$ One study showed that CA-125 titres $>1,000 \mathrm{U} / \mathrm{ml}$ correlated with malignancy but there was a reported case of peritoneal tuberculosis with a CA-125 level of $1,081 \mathrm{U} / \mathrm{ml}^{10}{ }^{10}$ So, no definite CA-125 level can differentiate malignant from non-malignant causes, but it can be used to monitor response to treatment. ${ }^{6,9}$

Tuberculin Skin Test is non-reliable as its positivity is not-specific for active tuberculosis and has low sensitivity with high false negative rate $(15 \%$ to $60 \%)$. Ascitic fluid analysis shows usually yellow citrine colour $(77 \%$ to $91 \%)$, protein content ( $>30$ g/l), lymphocyte-predominant TLC of $>400$ cells/ $\mathrm{ml}(68.3 \%$ of cases), ZN-staining for AFB $(<3 \%)$, cytological study for neoplastic cells, LDH assay ( $>90$ IU/1; Sn- $77 \%$ to $90 \%$ and Sp-14\%), Serum Amino-Acid Gradient $(<11 \mathrm{~g} / \mathrm{l}$; Sn $=100 \%$ but low $\mathrm{Sp}$ ), low glucose concentration. Due to too low specificity, LDH, SAAG and glucose assays are not recommended for its diagnosis. Other tests like Adenosine Deaminase Assay ( $\geq 30 \mathrm{U} / \mathrm{l}$; Sn $=96 \%$, $\mathrm{Sp}=98 \%)$, interferon- $\gamma(\geq 3.2 \mathrm{IU} / \mathrm{ml} ; \mathrm{Sn}=93 \%$ and $\mathrm{SP}=98 \%$ ), specific immunoglobulin (IgG antibody to $43 \mathrm{kDa}$ antigen of $\mathrm{MT} ; \mathrm{Sn}=100 \%$ and $\mathrm{Sp}=$ 95.7\%) and PCR analysis for MTB ( $\mathrm{Sn}=0 \%$ to $80 \%$ ) can be employed for its diagnosis, former being less expensive and easily reproducible..$^{5,10}$

Examination of ascitic fluid or image-guided percutaneous peritoneal biopsy may make a definitive diagnosis, but a gold standard diagnostic tool is tissue diagnosis either from biopsies of tubercles obtained with diagnostic laparoscopy ( $\mathrm{Sn}=93 \%$ and $\mathrm{Sp}=98 \%)$ or intraoperative frozen section. Ovarian cancer being a serious condition and preoperative diagnosis of peritoneal-pelvic TB difficult, performing laparotomy may be justifiable ${ }^{5}$

\section{CONCLUSIONS}

Peritoneal-pelvic tuberculosis should always be considered as one of the differential diagnosis in an adult woman who lives in TB endemic region and presents with a radiologically identifiable pelvic mass, ascites and raised CA-125 levels to prevent any inappropriate treatment.

\section{ACKNOWLEDGEMENT}

We would like to thank Dr. Indira Aacharya, Dr. Raju Kumar Gupta, Dr. Bibhushan Neupane, Dr. Neelima Bajracharya for their support. 
To cite this article: Oli PR, Malla RV; Karmacharya K. A case of peritoneal-pelvic tuberculosis with elevated CA-125; An enigma as ovarian cancer. MJSBH. 2019;18(2);60-3.

Conflict of Interest: None declared

\section{REFERENCES}

1. Government of Nepal Ministry of Health and Population, Department of Health Services. Annual Report Department of Health Services 2072/73 (2015/2016). Teku, Kathmandu: Government of Nepal Ministry of Health and Population, Department of Health Services: 2018. Chapter 4.7 Tuberculosis; p. 138-148.

2. Türkmen İÇ, Başsüllü N, Çomunoğlu C, Bağcı P, Aydın Ö, Çomunoğlu N, et al. Female genital system tuberculosis: a retrospective clinicopathological study of 1,548 cases in Turkish women. Arch Gynecol Obstet. 2012;286(2): 379-84.

DOI: $10.1007 / \mathrm{s} 00404-012-2281-\mathrm{y}$

3. Kasper D, Fauci A, Hauser S, Longo D, Jameson J, Loscalzo J. Harrison's principles of internal medicine, 19 th ed. New Delhi: McGraw-Hill; 2015. Chapter 202 Tuberculosis; p.1102-1122.

4. Ilhan AH, Durmuşoğlu F. Case report of a pelvic-peritoneal tuberculosis presenting as an adnexal mass and mimicking ovarian cancer, and a review of the literature. Infect Dis Obstet Gynecol. 2004;12(2):87-9.

DOI: $10.1080 / 10647440400003568$

5. Liu Q, Zhang Q, Guan Q, Xu JF, Shi QL. Abdominopelvic tuberculosis mimicking advanced ovarian cancer and pelvic inflammatory disease: a series of 28 female cases. Arch Gynecol Obstet. 2014;289(3):623-9.

DOI: $10.1007 / \mathrm{s} 00404-013-3034-2$

6. Koc S, Beydilli G, Tulunay G, Ocalan R, Boran N, Ozgul N, et al. Peritoneal tuberculosis mimicking advanced ovarian cancer: a retrospective review of 22 cases. Gynecologic oncology. 2006;103(2):565-9.

DOI: 10.1016/j.ygyno.2006.04.010

7. Xi X, Shuang L, Dan W, Ting H, Han MY, Ying C, et al. Diagnostic dilemma of abdominopelvic tuberculosis: a series of 20 cases. Journal of cancer research and clinical oncology. 2010;136(12):1839-44.

DOI: $10.1007 / \mathrm{s} 00432-010-0842-7$

8. Liu Q, Zhang Q, Guan Q, Xu JF, Shi QL. Abdominopelvic tuberculosis mimicking advanced ovarian cancer and pelvic inflammatory disease: a series of 28 female cases. Arch Gynecol Obstet. 2014;289(3):623-9.

DOI: $10.1007 / \mathrm{s} 00404-013-3034-2$

9. Gosein MA, Narinesingh D, Narayansingh GV, Bhim NA, Sylvester PA. Peritoneal tuberculosis mimicking advanced ovarian carcinoma: an important differential diagnosis to consider. BMC research notes. 2013;6(1):88.

DOI: $10.1186 / 1756-0500-6-88$

10. Mohattane H, Piette JC, Bogdan A, Auzeby A, Touitou Y, Godeau P. Specificity of CA 125 tumor marker. A study of 328 cases of internal medicine. Presse medicale. 1988;17(43):2287-91.

PMID: 2463618 